\title{
Avaliação do impacto das ações de controle vetorial da doença de Chagas através do inquérito sorológico em Mambaí/Buritinópolis, Goiás
}

\author{
Evaluation of the impact of vector control programs through serological \\ testing in Mambai/ Buritinópolis, Goiás State
}

\author{
Rosaura Peñaranda-Carrillo', Eliana Furtado Moreira², Antônio Carlos Silveira', \\ João Leite ${ }^{3}$, Márcio Costa Vinhaes ${ }^{3}$, Cleudson Castro e Vanize Macêdo ${ }^{1}$
}

\begin{abstract}
Resumo Em 1999, realizamos a avaliação do impacto das medidas de controle vetorial sobre a transmissão da doença de Chagas nas áreas endêmicas Mambaí e Bruritinópolis (GO). Após o recenseamento populacional foram realizados os inquéritos entomológico das unidades domiciliares e sorológico da população. As amostras de sangue foram coletadas por punção digital, em papel de filtro. O teste sorológico utilizado inicialmente para detectar anticorpos contra Trypanosoma cruzi foi a reação de imunofluorescência indireta (IFI) quantitativa com ponto de corte a diluição 1/20 e, os reagentes realizaram a reação de hemaglutinação indireta (HAI). A prevalência da IFI reagente foi 12,3\% (95\% IC: 11,5-13,2\%). Triatoma infestans não foi encontrado nas habitações. A ausência de infecção de indivíduos menores de 14 anos e a ausência de T. infestans no inquérito entomológico demonstra o sucesso do programa de controle da doença de Chagas nessas áreas, podendo ser considerada interrompida a transmissão vetorial.
\end{abstract}

Palavras-chaves: Doença de Chagas. Inquérito sorológico. Inquérito entomológico. Triatoma infestans. Transmissão vetorial. Brasil.

\begin{abstract}
In 1999, we performed serological and entomological surveys to evaluate the impact of vectorial control measures against transmission of Chagas' disease in the endemic area of Mambaí and Buritinópolis (GO). A census was undertaken of the population, after which the entomological survey was performed regarding the dwelling units and serological evaluation of the human population. Blood samples were collected by digital puncture in filter paper. The first serologic test performed to detect antibodies against Trypanosoma cruzi was the indirect immunofluorescence test (IFI) with 1/20 positive dilution as cut-off point and, positive samples were further evaluated with indirect hemagglutination reaction (HAI). The prevalence of positive IFI reactions was 12.3\% (95\%CL: 11.5-13.2). Triatoma infestans was not found within the dwellings. The absence of infection among individuals younger than 14 years and, the absence of $\mathrm{T}$. infestans during the last entomological survey demonstrates the success of the control program of Chagas' disease in the studied area where the vectorial transmission can be considered to have been interrupted.
\end{abstract}

Key-words: Chagas' disease. Serologic survey. Entomologic survey. Brazil. Triatoma infestans. Vectorial transmission.

Em 1974, o Núcleo de Medicina Tropical da Universidade de Brasília iniciou em Mambaí, município do Estado de Goiás, um estudo clínico e epidemiológico da doença de Chagas. Neste ano, foi feito o levantamento geográfico da localidade e o recenseamento populacional de 4.252 habitantes ${ }^{4}{ }^{15}$. No ano seguinte, teve inicio a avaliação clínica, sorológica, radiológica, e eletrocardiográfica, sendo examinados 3.140 indivíduos. Simultaneamente, realizouse o levantamento triatomínico em, aproximadamente, 900 unidades domiciliares (UDs) ${ }^{41516}$.

A sorologia para a doença de Chagas foi realizada pelas reações de fixação do complemento, hemaglutinação indireta e imunofluorescência indireta (as três reações foram realizadas no Instituto de Medicina Tropical de São Paulo e no Instituto Mario

1. Núcleo de Medicina Tropical da Universidade de Brasília, Brasília, DF. 2. Fundação Ezequiel Dias, Belo Horizonte, MG. 3. Fundação Nacional de Saúde, Brasília, DF

Endereço para correspondência: Dra Vanize Macêdo. NMT/UnB. Caixa Postal 4517, 70919-970, Brasília, DF.

e-mail: tropical@unb.br

Recebido para publicação em 5/7/2001. 
Fatala Chaben, em Buenos Aires). A prevalência de sorologia reagente para esta doença foi $34,4 \%$ sendo $17,9 \%$ em crianças entre zero e dez anos de idade ${ }^{345}$.

No levantamento triatomínico, ficou demonstrado que $54 \%$ das UDs estavam infestadas pelo Triatoma infestans. Em 1979, foram novamente pesquisadas 455 dessas UDs e observou-se aumento da infestação intradomiciliar para $74 \%{ }^{19}$.

A partir de 1980, foi iniciado pela Superintendência de Campanhas de Saúde Pública (SUCAM/Ministério da Saúde) o tratamento químico dos domicílios com inseticida (hexacloreto de benzeno-BHC), com cobertura integral da área. Logo a seguir, foram implantadas as atividades de vigilância epidemiológica, tendo a participação das equipes da UnB e da SUCAM, com o envolvimento da população78101518.

Essas atividades de vigilância epidemiológica consistiam basicamente em notificação, pelo morador, da presença de triatomíneos em sua casa, seguida de captura manual pela técnica hora/homem, feita pelo agente de saúde pública devidamente treinado, com posterior uso de insetífugo (pirisa líquida). Esse trabalho era acompanhado pela inspeção das caixas de Gómez Nuñez e de calendários, buscando a identificação de vestígios e a coleta de triatomíneos em bolsas plásticas que acompanhavam os calendários. Quando comprovada a infestação, a unidade domiciliar era devidamente tratada com inseticida de ação residual $(\mathrm{BHC})^{79101718}$.
A avaliação da eficácia do programa, no período de 1980 a 1984, mostrou, neste último ano, que a taxa de infestação intradomiciliar pelo $T$. infestans diminuiu significativamente (28,6\% para $14,2 \%)$, mas a infestação peridomiciliar pelo $T$. sordida se manteve em $32 \%{ }^{10}$.

Em 1986-87, realizou-se um novo recenseamento populacional neste município, constatando-se que a população aumentou para 6.795 habitantes. Em 1.955 destes indivíduos, foi realizada sorologia para doença de Chagas pelas reações de imunofluorescência indireta (IFI), hemaglutinação indireta (HAI) e imunoabsorvência ligada a enzimas (ELISA). A prevalência encontrada de reação sorológica reagente foi $9,9 \%$. Das 715 crianças na faixa etária de zero a dez anos que participaram deste inquérito $2,2 \%$ foram soro reagentes para a doença de Chagas ${ }^{3}$.

Depois da borrifação em massa (1980), realizaramse expurgos seletivos das casas, acrescidos da vigilância feita pela população. Com isso, a taxa de infestação domiciliar caiu para zero por cento e, como conseqüência, a prevalência de infecção chagásica na população também diminuiu ${ }^{810}$.

O inquérito sorológico tem sido o procedimento individual mais valioso como indicador de infecção humana 352223 . Por este motivo, através deste, se propôs avaliar o impacto das ações de controle de vetores na transmissão da doença de Chagas nos municípios de Mambaí e Buritinópolis, áreas de estudo longitudinal, mantidas a longo tempo sob vigilância, com participação comunitária.

\section{MATERIAL E MÉTODOS}

Este trabalho é parte do projeto Avaliação Epidemiológica do Impacto das Ações de Controle Vetorial da Doença de Chagas em Mambaíl Buritinópolis, Goiás. É um estudo de prevalência, onde foram avaliadas as populações dos municípios de Mambaí e Buritinópolis (antigo distrito de Buritis, desmembrado do município de Mambaí, em 29 de abril de 1992, pela Lei estadual №11.705), entre os meses de agosto a dezembro de 1999. Simultaneamente ao recenseamento da população, foi realizado o inquérito sorológico para doença de Chagas em 69,27\% da população e, o entomológico, em $100 \%$ das UDs, tanto da zona urbana quanto da rural.

Recenseamento. Foi realizado por guardas sanitários da Fundação Nacional de Saúde (FUNASA), devidamente treinados. Depois de mapeadas as localidades, foram feitas visitas domiciliares em que se preencheu uma ficha familiar que incluía: município, endereço, nome do chefe da família e demais integrantes por ordem descendente, idade e sexo de cada um dos integrantes da família. Através do recenseamento, realizado em 1974-1975, pelo Núcleo de Medicina Tropical/Universidade de Brasília, foram identificados os indivíduos cadastrados no Projeto Mambaí naquela época.
Inquérito entomológico. A pesquisa entomológica foi realizada por técnicos da FUNASA, através da busca ativa, pela técnica hora/homem, com tempo controlado de uma hora por UD, fazendo-se a captura com o uso de pinça entomológica. Os exemplares capturados foram classificados segundo a espécie e o estádio de desenvolvimento, e submetidos ao exame a fresco do conteúdo intestinal. Para o estudo de fontes alimentares, analisou-se o conteúdo estomacal dos triatomíneos através da técnica de precipitinas em tubos capilares ${ }^{1314} 21$.

Inquérito sorológico. O inquérito sorológico incluiu as pessoas residentes nos dois municípios que se encontravam nas unidades domiciliares no momento da visita do guarda sanitário. Foram explicados aos integrantes da família os procedimentos a serem realizados e a importância do exame sorológico para a doença de Chagas. De cada indivíduo solicitou-se o consentimento verbal. Para os menores de idade, o consentimento foi dado pelos pais ou responsáveis. O projeto foi aprovado pelo Comitê de Ética da Faculdade de Medicina da Universidade de Brasília, como parte do estudo longitudinal da doença de Chagas em Mambaí/Buritinópolis.

As amostras de sangue foram coletadas por punção digital, com lancetas descartáveis, em papel de filtro, em duas alíquotas de aproximadamente $20 \mathrm{~mm}$ de 
diâmetro, distribuídas em tira de papel com dimensões de $7,5 \times 2,5 \mathrm{~cm}$. As tiras foram intercaladas com papel celofane, isolando-se as amostras, e identificadas com um número de registro ${ }^{11}$. Para cada indivíduo, foi preenchida uma ficha com o número de registro, nome, idade, sexo, município de residência, endereço e município de nascimento.

Os guardas sanitários entregavam o material coletado, a cada sete dias, a um dos pesquisadores que permanecia no posto médico da Universidade de Brasília localizado na cidade de Mambaí. Posteriormente, as amostras eram checadas com as fichas que continham os dados pessoais de cada indivíduo. As amostras de sangue foram acondicionadas em caixas de papelão e mantidas à temperatura ambiente, sendo logo enviadas ao Núcleo de Medicina Tropical/Universidade de Brasília para serem encaminhadas ao laboratório de imunologia da
Fundação Ezequiel Dias/MG, onde foram realizados os exames sorológicos para a pesquisa de infecção pelo T. cruzi.

Inicialmente, realizou-se a triagem sorológica através da reação de IFI quantitativa, tendo como ponto de corte a diluição 1:20. Todas as amostras reagentes foram tituladas e submetidas à reação sorológica de $\mathrm{HAl}^{3}$.

Nos indivíduos menores de 20 anos de idade, com testes reagentes, quer seja IFI ou HAI, foram coletados $10 \mathrm{ml}$ de sangue, por punção venosa com seringa descartável, para a realização de sorologia para doença de Chagas pelas reações de IFI, HAI e ELISA. Os indivíduos sororreagentes pelas três reações foram investigados para avaliação da autoctonia da infecção tripanossômica.

Naqueles indivíduos com infecção recente, foi indicado tratamento com droga tripanossomicida.

\section{RESULTADOS}

Recenseamento. O número total de indivíduos recenseados foi 8.143 , sendo que 4.692 (57,6\%) residiam em Mambaí e 3.451 (42,4\%) em Buritinópolis. Em Mambaí,
$54,4 \%$ da população moravam na área urbana e, $45,6 \%$ na área rural, enquanto a maioria $(58 \%)$ da população de Buritinópolis vivia na área rural (Tabela1).

Tabela 1- Recenseamento de acordo com o município e a área de residência. Mambaíl Buritinópolis (GO), 1999.

\begin{tabular}{|c|c|c|c|c|c|c|}
\hline \multirow[b]{2}{*}{ Município } & \multicolumn{3}{|c|}{ Área urbana } & \multicolumn{2}{|c|}{ Área rural } & \multirow{2}{*}{$\begin{array}{c}\text { Total } \\
\%\end{array}$} \\
\hline & $\mathrm{n}^{0}$ & $\%$ & $\mathrm{n}^{0}$ & $\%$ & $\mathrm{n}^{0}$ & \\
\hline Mambaí & 2.551 & 54,4 & 2.141 & 45,6 & 4.692 & 57,6 \\
\hline Buritinópolis & 1.448 & 42,0 & 2.003 & 58,0 & 3.451 & 42,4 \\
\hline Total & 3.999 & 49,1 & 4.144 & 50,9 & 8.143 & 100,0 \\
\hline
\end{tabular}

Inquérito entomológico. Das 67 localidades existentes e pesquisadas, em 48 comprovou-se a presença de triatomíneos, com taxa de dispersão de $71,6 \%$.

A infestação foi marcadamente peridomiciliar. Os índices de infestação peridomiciliar em Mambaí correspondem a $8,7 \%$ e em Buritinópolis 12,1\%, com taxas para o intradomicílio de $0,7 \%$ e $1,2 \%$ respectivamente.

T. sordida foi a espécie identificada em $97,3 \%$ das capturas, sendo, ainda, a única encontrada no intradomicílio, com alguns poucos exemplares, e a única na qual se comprovou infecção natural pelo $T$. cruzi. As aves constituíram-se na fonte alimentar mais freqüente (45\%) do T. sordida e, apenas $2,8 \%$ das reações foram positivas para anti-soro humano.

O resultado mais significativo, no entanto, no que diz respeito à pesquisa entomológica propriamente dita, foi um dado negativo. A completa ausência de $T$. infestans nos dois municípios.

Inquérito Sorológico. Foram coletadas e examinadas 5.642 amostras de sangue, o que representa $69,3 \%$ da população recenseada, da qual 58,5\% (3.302) morava em Mambaí e 41,5\% (2.340) em Buritinópolis. A maioria $(52,6 \%)$ residia em área rural.
Do total das amostras coletadas e examinadas, $53,8 \%$ pertenciam a indivíduos do sexo feminino e $46,2 \%$ do sexo masculino. A média de idade foi $24,8 \%$, variando de zero a 97 anos; $51,7 \%$ (2.922 pessoas) eram menores de 20 anos de idade (Tabela 2).

A maioria $(69,3 \%)$ da população era autóctone, sendo $48,1 \%$ de Mambaí, $21,2 \%$ de Buritinópolis e, $30,7 \%$ dos municípios limítrofes.

A prevalência de exames sorológicos reagentes para a doença de Chagas, segundo a reação de IFI, com títulos iguais ou superiores a 1/20, foi 12,3\% (695/5642), com intervalo de $95 \%$ de confiança entre $11,5 \%$ a $13,2 \%$ (Tabela 3).

Dentre a população em que foi coletada amostra de sangue em papel de filtro, $2.922(51,7 \%)$ eram menores de 20 anos de idade e, desses, só $18(0,6 \%)$ indivíduos (95\% IC: 0,37-0,99) tinham reação de IFI reagente (Tabela 3).

As 695 amostras reagentes para a IFI foram submetidas à reação de HAl para doença de Chagas, encontrando-se $472(67,9 \%)$ reagentes, $200(28,8 \%)$ não-reagentes e $22(3,2 \%)$ amostras com resultados indeterminados (Tabela 4).

Considerando-se as reações sorológicas positivas, tanto para a reação de $\mathrm{IFI}$, quanto para a de $\mathrm{HAI}$, a 
Tabela 2 - População do inquérito sorológico para a doença de Chagas segundo a faixa etária e o sexo, Mambaí/Buritinópolis, 1999.

\begin{tabular}{lccrrr}
\hline & \multicolumn{2}{c}{ Sexo } & & \multicolumn{2}{c}{ Total } \\
\cline { 2 - 3 } \cline { 6 - 6 } Faixa etária & feminino & masculino & & $\mathrm{n}^{\circ}$ & $\%$ \\
\hline$<1$ & 40 & 46 & 86 & 1,5 \\
$1-4$ & 284 & 317 & 601 & 10,7 \\
$5-9$ & 408 & 390 & & 798 & 14,1 \\
$10-14$ & 422 & 392 & 814 & 14,4 \\
$15-19$ & 334 & 289 & 623 & 11,0 \\
$20-29$ & 437 & 307 & 744 & 13,2 \\
$30-39$ & 417 & 282 & 699 & 12,4 \\
$40-49$ & 293 & 215 & 508 & 9,0 \\
$50-59$ & 199 & 148 & 347 & 6,2 \\
$\geq 60$ & 204 & 218 & & 422 & 7,5 \\
\hline Total $(\%)$ & $3.038(58,8)$ & $2.604(46,2)$ & $5.642(100,0)$ & 100,0 \\
\hline
\end{tabular}

Tabela 3- Reação de imunofluorescência indireta para a doença de Chagas, de acordo com os títulos dos indivíduos estudados e a faixa etária. Mambaí/Buritinópolis (GO), 1999.

\begin{tabular}{|c|c|c|c|c|c|c|c|c|}
\hline \multirow[b]{2}{*}{ Faixa etária } & \multicolumn{7}{|c|}{ Imunofluorescência Indireta } & \multirow[b]{2}{*}{ Total } \\
\hline & $<1 / 20$ & $1 / 20$ & $1 / 40$ & $1 / 80$ & $1 / 160$ & $1 / 320$ & $1 / 640$ & \\
\hline$<1$ & 84 & 1 & 0 & 1 & 0 & 0 & 0 & 86 \\
\hline $1-4$ & 601 & 0 & 0 & 0 & 0 & 0 & 0 & 601 \\
\hline $5-9$ & 795 & 0 & 1 & 0 & 1 & 1 & 0 & 798 \\
\hline $10-14$ & 812 & 0 & 1 & 1 & 0 & 0 & 0 & 814 \\
\hline $15-19$ & 612 & 2 & 5 & 4 & 0 & 0 & 0 & 623 \\
\hline $20-29$ & 673 & 8 & 28 & 26 & 7 & 2 & 0 & 744 \\
\hline $30-39$ & 521 & 14 & 65 & 71 & 25 & 3 & 0 & 699 \\
\hline $40-49$ & 353 & 14 & 47 & 69 & 19 & 5 & 1 & 508 \\
\hline $50-59$ & 217 & 13 & 40 & 47 & 19 & 9 & 2 & 347 \\
\hline$\geq 60$ & 279 & 13 & 32 & 49 & 38 & 9 & 2 & 422 \\
\hline Total & 4.947 & 65 & 219 & 268 & 109 & 29 & 5 & 5.642 \\
\hline Porcentagem & 87,7 & 1,2 & 3,9 & 4,8 & 1,9 & 0,5 & 0,1 & 100,0 \\
\hline
\end{tabular}

\begin{tabular}{|c|c|c|c|c|c|}
\hline \multirow[b]{2}{*}{ IFI } & \multicolumn{4}{|c|}{ Hemaglutinação indireta } & \multirow[b]{2}{*}{ Total } \\
\hline & reagente & indeterminado & não reagente & não realizado & \\
\hline $1 / 20$ & 15 & 4 & 45 & 1 & 65 \\
\hline $1 / 40$ & 132 & 8 & 79 & 0 & 219 \\
\hline $1 / 80$ & 194 & 8 & 66 & 0 & 268 \\
\hline $1 / 160$ & 99 & 2 & 8 & 0 & 109 \\
\hline $1 / 320$ & 27 & 0 & 2 & 0 & 29 \\
\hline $1 / 640$ & 5 & 0 & 0 & 0 & 5 \\
\hline Total & 472 & 22 & 200 & 1 & 695 \\
\hline Porcentagem & 67,9 & 3,2 & 28,8 & 0,1 & 100,0 \\
\hline
\end{tabular}

prevalência para a doença de Chagas foi 8,37\% (95\% IC: 7,7-9,12).

A maioria da população $(76,6 \%)$ com resultado da reação de IFI igual ou maior que 1/20 era autóctone, sendo $16,8 \%$ dos examinados procedentes de municípios limítrofes: Alvorada do Norte, Posse e Simolandia (G0), Correntina, Cocos (BA) (Tabela 5).

Quatrocentos e quarenta e nove $(64,6 \%)$ pessoas com IFI reagente moravam no município de Buritinópolis, sendo 71,5\% na área rural (Tabela 6).
Foram identificados 791 indivíduos do Projeto Mambaí dos quais, desde 1975, conheciam-se os resultados da sorologia para a doença de Chagas. Dos 344 indivíduos sororeagentes em 1975, 44 (9,7\%) tiveram reação IFI não-reagente em 1999. Dos 435 com testes sorológicos não-reagentes em 1975, 33 (9,7\%) foram soro reagentes à IFI no inquérito atual. Doze indivíduos tinham resultados sorológicos duvidosos em 1975 e, desses, a metade apresentou IFI reagente e, a outra metade IFI não-reagente (Tabela 7). 
Tabela 5- Reação de IFI com títulos iguais ou maiores a 1/20, segundo o município de procedência

\begin{tabular}{lrr}
\hline \multirow{2}{*}{ Município de Procedência } & \multicolumn{2}{c}{ IFI (título = 1/20) } \\
\cline { 2 - 3 } Mambaí & $\mathrm{n}^{\circ}$ & $\%$ \\
Buritinópolis & 284 & 40,9 \\
Correntina-Cocos (BA) & 69 & 35,7 \\
Posse-Alvorada-Simolandia (GO) & 48 & 9,9 \\
Outros municípios de Goiás & 31 & 6,9 \\
Municípios de MG & 11 & 4,5 \\
Outros municípios & 4 & 1,6 \\
\hline Total & 695 & 0,5 \\
\hline
\end{tabular}

Tabela 6- Reação de IFI com títulos iguais ou maiores a 1/20, segundo o município e a área de residência.

\begin{tabular}{|c|c|c|c|c|c|c|}
\hline \multirow[b]{4}{*}{ Município de residência } & \multirow{2}{*}{\multicolumn{4}{|c|}{$\begin{array}{l}\mathrm{IFI} \text { (títulos }=1 / 20 \text { ) } \\
\text { área de residência }\end{array}$}} & & \\
\hline & & & & & & \\
\hline & \multicolumn{2}{|c|}{ rural } & \multicolumn{2}{|c|}{ urbana } & \multicolumn{2}{|c|}{ Total } \\
\hline & $\mathrm{n}^{0}$ & $\%$ & $\mathrm{n}^{0}$ & $\%$ & $\mathrm{n}^{0}$ & $\%$ \\
\hline Mambaí & 173 & 70,3 & 73 & 29,7 & 246 & 35,4 \\
\hline Buritinópolis & 324 & 72,1 & 125 & 27.9 & 449 & 64,6 \\
\hline Total & 497 & 71,5 & 198 & 28,5 & 695 & 100,0 \\
\hline
\end{tabular}

Tabela 7- Correlação dos resultados dos exames sorológicos para a doença de Chagas de 1975 com o resultado de IFI realizada em 1999.

\begin{tabular}{|c|c|c|c|c|c|c|}
\hline \multirow{3}{*}{$\begin{array}{l}\text { Exames sorológicos } \\
\text { para DC de } 1975^{\star}\end{array}$} & \multicolumn{4}{|c|}{ Reação de IFI realizada em 1999} & & \\
\hline & \multicolumn{2}{|c|}{ reagente } & \multicolumn{2}{|c|}{ não-reagente } & \multicolumn{2}{|c|}{ Total } \\
\hline & $\mathrm{n}^{0}$ & $\%$ & $\mathrm{n}^{0}$ & $\%$ & $\mathrm{n}^{0}$ & $\%$ \\
\hline Reagente & 300 & 88,5 & 44 & 9,7 & 344 & 43,5 \\
\hline Não-reagente & 33 & 9,7 & 402 & 89,0 & 435 & 55,0 \\
\hline Duvidosa & 6 & 1,8 & 6 & 1,3 & 12 & 1,5 \\
\hline Total & 339 & 100,0 & 452 & 100,0 & 791 & 100,0 \\
\hline
\end{tabular}

* Sorologia para a doença de Chagas em 1975 realizada através das reações de Fixação do Complemento, IFI e HAI.

A maioria $(58,7 \%)$ dos indivíduos com IFI reagente era do sexo feminino. Não houve diferença estatística quanto ao sexo entre indivíduos menores de 20 anos de idade (Tabela 8).
Nos 18 indivíduos menores de 20 anos de idade, com reação de IFI reagente em papel de filtro, foram repetidos os exames sorológicos para doença de Chagas (IFI, HIA e ELISA) de sangue coletado por

Tabela 8- Reação de IFI com títulos iguais ou maiores a 1/20, segundo a faixa etária e o sexo, Mambaí/Buritinópolis, 1999.

\begin{tabular}{lccc}
\hline & \multicolumn{2}{c}{ Imunofluorescência indireta (título $\geq 1 / 20)$} & \\
\cline { 2 - 3 } Faixa etária & feminino & masculino & Total \\
\hline$<1$ & 1 & 1 & 2 \\
$1-4$ & 0 & 0 & 0 \\
$5-9$ & 1 & 2 & 3 \\
$10-14$ & 2 & 0 & 2 \\
$15-19$ & 5 & 6 & 11 \\
$20-29$ & 38 & 33 & 71 \\
$30-39$ & 113 & 65 & 178 \\
$40-49$ & 97 & 58 & 155 \\
$50-59$ & 76 & 54 & 130 \\
${ }^{3} 60$ & 75 & 68 & 143 \\
\hline Total & 408 & 287 & 695 \\
Porcentagem & 58,7 & 41,3 & 100,0 \\
\hline
\end{tabular}


punção venosa. Em três dessas pessoas não foi possível a realização desses exames sorológicos (Tabela 9).
Um indivíduo de 19 anos teve resultados nãoreagentes aos testes de IFI, HAI e ELISA. Dois indivíduos, um de 14 e outro de 17 anos, apresentaram

\begin{tabular}{|c|c|c|c|c|c|c|c|}
\hline \multirow[b]{2}{*}{ Indivíduos $\left(\mathrm{n}^{\circ}\right)$} & \multirow[b]{2}{*}{ Idade (anos) } & \multirow[b]{2}{*}{ Autoctonia } & \multicolumn{2}{|c|}{$\begin{array}{l}\text { Exames sorológicos em amostras } \\
\text { em papel de filtro }\end{array}$} & \multicolumn{3}{|c|}{$\begin{array}{c}\text { Exames sorológicos em sangue } \\
\text { de punção venosa }\end{array}$} \\
\hline & & & IFI & $\mathrm{HAl}$ & IFI & $\mathrm{HAl}$ & ELISA \\
\hline 1 & 0 & Sim & $1 / 40$ & $\mathrm{R}^{*}$ & - & - & - \\
\hline 2 & 0 & Sim & $1 / 20$ & $\mathrm{NR}^{* *}$ & NR & NR & NR \\
\hline 3 & 5 & Sim & $1 / 40$ & NR & NR & NR & NR \\
\hline 4 & 6 & Sim & $1 / 160$ & $\mathrm{R}$ & NR & NR & NR \\
\hline 5 & 9 & Sim & $1 / 320$ & NR & NR & NR & NR \\
\hline 6 & 10 & Sim & $1 / 160$ & $\mathrm{R}$ & NR & NR & NR \\
\hline 7 & 14 & Sim & $1 / 40$ & $\mathrm{R}$ & $1 / 80$ & IND ${ }^{\star \star \star}$ & $\mathrm{R}$ \\
\hline 8 & 15 & Não & $1 / 80$ & $\mathrm{R}$ & $1 / 160$ & $\mathrm{R}$ & $\mathrm{R}$ \\
\hline 9 & 15 & Não & $1 / 20$ & $\mathrm{R}$ & $1 / 160$ & $\mathrm{R}$ & $\mathrm{R}$ \\
\hline 10 & 17 & Sim & $1 / 20$ & NR & $1 / 80$ & IND & $\mathrm{R}$ \\
\hline 11 & 17 & Sim & $1 / 80$ & $\mathrm{R}$ & $1 / 80$ & $\mathrm{R}$ & $\mathrm{R}$ \\
\hline 12 & 17 & Não & $1 / 40$ & NR & $1 / 160$ & $\mathrm{R}$ & $\mathrm{R}$ \\
\hline 13 & 17 & Sim & $1 / 40$ & $\mathrm{R}$ & - & - & - \\
\hline 14 & 17 & Não & $1 / 40$ & $\mathrm{R}$ & - & - & - \\
\hline 15 & 19 & Sim & $1 / 40$ & $\mathrm{R}$ & $1 / 80$ & $\mathrm{R}$ & $\mathrm{R}$ \\
\hline 16 & 19 & Sim & $1 / 40$ & $\mathrm{R}$ & $1 / 80$ & $\mathrm{R}$ & $\mathrm{R}$ \\
\hline 17 & 19 & Sim & $1 / 80$ & $\mathrm{R}$ & NR & NR & NR \\
\hline 18 & 19 & Sim & $1 / 80$ & $\mathrm{R}$ & $1 / 160$ & $\mathrm{R}$ & $\mathrm{R}$ \\
\hline
\end{tabular}

IFI e ELISA reagentes, mas o resultado da $\mathrm{HAI}$ foi indeterminado e em sete indivíduos foi confirmada a infecção chagásica pelas três reações sorológicas (IFI, HAI e ELISA).

Vale a pena ressaltar que apenas seis $(0,3 \%)$ crianças (95\% IC: 0,11-0,64) menores de 14 anos (38,1\% da população, de 2.149 pessoas) tiveram IFI em papel de filtro para a doença de Chagas reagente; contudo, esta sorologia não foi confirmada pelos exames de IFI, HAI e ELISA realizados em amostra de sangue coletada por punção venosa.

Quatorze desses indivíduos eram autóctones dos municípios do estudo, dois eram da Bahia, um de Damianópolis (GO) e um de Minas Gerais (Tabela 9).

\section{DISCUSSÃO}

Mambaí e Buritinópolis são municípios do estado de Goiás nos quais vêm sendo desenvolvidos estudos clínicos e epidemiológicos da doença de Chagas desde 1975. As medidas de controle vetorial iniciaram-se em 1980 através da borrifação em massa e, posteriormente, com expurgos seletivos, isto somado à vigilância feita pela população. Com este trabalho, avaliamos o impacto que tiveram essas medidas de controle na infecção humana pelo T. cruzi.

$\mathrm{O}$ recenseamento realizado pela FUNASA junto à Universidade de Brasília nos municípios do estudo mostrou um crescimento populacional de aproximadamente $20 \%$ em relação à existente em 1987, e de $90 \%$ em relação à população recenseada em $1975^{34}$.

A soroprevalência para a doença de Chagas de $34,4 \%$, verificada no inquérito de $1975^{4}$, caiu para 12,3\% em 1999.
A analise do comportamento da infecção chagásica nas populações dos Municípios de Mambaí e de Buritinópolis, em relação a três momentos do Projeto Mambaí, mostrou uma redução importante da prevalência de sorologias reagentes para a doença de Chagas na faixa etária de zero a dez anos de idade. Em 1975, a prevalência da infecção chagásica em crianças nesta faixa etária era 17,9\%; em 1987, sete anos após a implantação das medidas de vigilância epidemiológica, essa prevalência caiu para $2,2 \%$, sendo que, neste último inquérito (1999), não mais se constatou infecção chagásica em crianças entre zero e dez anos de idade ${ }^{34}$.

Os resultados das reações de IFI, HAI e ELISA, realizadas em sangue coletado por punção venosa em $18(0,6 \%)$ indivíduos menores de 20 anos, mostrou que não mais existe transmissão vetorial da doença de Chagas em Mambaí/Buritinópolis após 13 anos de 
controle vetorial, uma vez que em nenhum indivíduo nesta faixa etária foi comprovada a infecção pelo $T$. cruzi. Da mesma forma, foi demonstrado não ter havido transmissão congênita, considerando que a sorologia na faixa de zero a dez anos foi não-reagente.

As técnicas sorológicas (IFI, HAI e ELISA) classicamente empregadas para estudos de tipo epidemiológico, utilizadas nesta pesquisa, apresentam boa sensibilidade, próxima a $100 \%$, tanto em soro quanto em eluatos, mas todas elas têm baixa especificidade, podendo apresentar índices variáveis de reações falso positivas ${ }^{161220}$. Isso provavelmente poderia explicar a divergência dos resultados encontrados neste inquérito com os resultados dos mesmos indivíduos, realizados em 1975, pelo Projeto Mambai ${ }^{36}$. Igualmente, a discordância de resultados quando se realizam os mesmos testes sorológicos em laboratórios diferentes tem sido com freqüência relatada na literatura ${ }^{356}$.

As ações de controle vetorial ocasionaram um grande impacto na diminuição da prevalência da doença de Chagas, através da interrupção da transmissão. Isto ficou bem demonstrado nas ações de controle do T. infestans com participação comunitária, realizadas nos municípios de Mambaí/Buritinópolis, alcançando a interrupção da transmissão vetorial da doença de Chagas pelo $T$. infestans, após treze anos de vigência do programa de controle.

Torna-se necessária a manutenção de medidas de vigilância epidemiológica permanente para que seja mantido o sucesso do programa, garantindo-se, assim melhores condições de saúde para estas comunidades.

\section{REFERÊNCIAS BIBLIOGRÁFICAS}

1. Camargo ME, Hoshino-Shimizu S, Macêdo V, Peres BA, Castro C. Diagnóstico sorológico da infecção humana pelo Trypanosoma cruzi estudo comparativo de testes de fixação do complemento, imunofluorescência, hemaglutinação e floculação, em 3.624 soros. Revista do Instituto de Medicina Tropical de São Paulo 19: 254-260, 1977.

2. Camargo ME, Silva GR, Castilho EA, Silveira AC. Inquérito Sorológico da Prevalência de Infecção Chagásica no Brasil, 1975/ 1980. Revista do Instituto de Medicina Tropical de São Paulo 26: 192-204, 1984.

3. Castillo EAS. Estudo da infecção chagásica na população nascida após implantação do programa de controle do Triatoma infestans no município de Mambaí - Goiás. Tese de Mestrado. Universidade de Brasília, Brasilía, DF, 1989.

4. Castro CN. Influência da parasitemia no quadro clínico da doença de Chagas. Revista de Patologia Tropical 9: 73-136, 1980.

5. Castro CN, Camargo M, Cerisola JA, Macêdo V, Prata A. Prevalência sorológica da infecção chagásica em Mambaí (GO), no ano de 1976. Revista da Sociedade Brasileira de Medicina Tropical 19 (sup II): 48, 1986.

6. Fuchs AD, Fioratti VL, Mello VA, Boainain E. Diagnóstico sorológico na doença de Chagas estudo comparativo de diferentes técnicas. Revista do Instituto de Medicina Tropical de São Paulo 22: 242-245, 1980.

7. Garcia-Zapata MT. Vigilância epidemiológica no controle do Triatoma infestans em duas áreas no estado de Goiás-Brasil. Tese de Mestrado. Universidade de Brasília, Brasília, 1985.

8. Garcia-Zapata MT, Marsden PD. Control of the transmission of Chagas disease in Mambaí, Goias, Brazil (1980-1988). The American Journal of Tropical Medicine and Hygiene 46: 440-443, 1992.

9. Garcia-Zapata MT, Marsden PD. Enfermedad de Chagas: contro y vigilancia con insecticidas y participacion comunitaria en Mambaí, Goias, Brasil. Boletín de la Oficina Sanitaria Panamericana 116: 97-110, 1994

10. Garcia-Zapata MT, Marsden PD, Virgens D, Penna R, Soares V, Brasil IA, Castro CN, Prata A, Macêdo V. O controle da Transmissão da Doença de Chagas em Mambaí Goiás, Brasil (1982-1984). Revista da Sociedade Brasileira de Medicina Tropical 19: 219-225, 1986.
11. Guimarães MCS. Coleta de sangue em papel de filtro para fins de inquéritos soroepidemiológicos. Revista da Sociedade Brasileira de Medicina Tropical 17: 45, 1984.

12. Kagan IG, Goldsmith RS, Zarete-Castaneda R, Allain DS. Evaluación de pruebas serológicas utilizadas para estudiar la enfermedad de Chagas. Boletin de la Oficina Sanitaria Panamericana 87: 309-317, 1979.

13. Lorosa ES, Andrade RE, Santos SM e Pereira CA. Estudo da infecção natural e da fonte alimentar do Triatoma sordida (STAL, 1859), (Hemíptera-Reduviidae) na região norte de Minas Gerais, Brasil, através da reação de Precipitina. Entomologia y Vectores 5: 13-22, 1998.

14. Lorosa ES, Andrade RE, Santos SM, Pereira CA, Vinhaes MC. Estudo do comportamento alimentar de algumas espécies de triatomineos com auxilio da tecnica de precipitina. Entomologia y Vectores 6:112-125, 1999.

15. Marsden PD. The control of Chagas' Disease in Mambaí, Brazil: the initial phases. Infection Control 2:466-470, 1981.

16. Marsden PD, Garcia-Zapata MT, Castillo EAS, Prata A, Macêdo V. Los 13 primeros años del control de la enfermedad de Chagas en Mambaí, Goiás, Brasil, 1980-1992. Boletín de la Oficina Sanitaria Panamericana 116:111-117, 1994.

17. Marsden PD, Penna R. A 'vigilance unit' for households subject to triatominae control. Transactions of the Royal Society of Tropical Medicine and Hygiene 76:790-792, 1982.

18. Marsden PD, Virgens D, Castro CN, Brasil IP, Ferreira R, Silveira AC, Matos CA, Macedo V, Prata A. The Control of Chagas' Disease Transmission in Mambaí, Goiás-Brazil (1980-1981). Revista da Sociedade Brasileira de Medicina Tropical 16:189195, 1983.

19. Marsden PD, Virgens D, Magalhães I, Tavares-Neto J, Pereira R, Costa $\mathrm{CH}$, Castro CN, Macêdo V, Prata A. Ecologia doméstica do Triatoma infestans em Mambaí, Goiás-Brasil. Revista do Istituto de Medicina Tropical de São Paulo 24:364-373, 1982.

20. Prata A, Mayrink W, Sofré AG, Almeida JA. Discrepâncias relativas entre resultados de reações de Guerreiro-Machado executadas entre três diferentes laboratórios. Revista de Patologia Tropical 4: 35-38, 1975. 
21. Siqueira AF. Estudo sobre a reação da precipitina aplicada a identificação de sangue ingerido por triatomíneos. Revista do Instituto de Medicina Tropical de São Paulo 2: 41-53, 1960.

22. Souza SL, Camargo ME. The use of filter paper blood smears in a practical fluorescent test for american trypanosomiasis serodiagnosis. Revista do Instituto de Medicina Tropical de São Paulo 8:255-258, 1966.

23. Zicker F, Oliveira M, Luquetti AO, Oliveira OS, Smith PG. Seroprevalence of Trypanosoma cruzi infection among unskilled urban workers in central Brazil. Transactions of the Royal Society of Tropical Medicine and Hygiene 83:511-513, 1989. 